\title{
VASE: Volume-Aware Surface Evolution for Surface Reconstruction from Incomplete Point Clouds
}

\author{
Andrea Tagliasacchi ${ }^{1}$, Matt Olson ${ }^{1}$, Hao Zhang $^{1}$, Ghassan Hamarneh ${ }^{1}$, and Daniel Cohen-Or ${ }^{2}$ \\ ${ }^{1}$ GrUVi lab, School of Computing Science, Simon Fraser University, Canada \\ ${ }^{2}$ School of Computer Science, Tel Aviv University, Israel
}

\begin{abstract}
Objects with many concavities are difficult to acquire using laser scanners. The highly concave areas are hard to access by a scanner due to occlusions by other components of the object. The resulting point scan typically suffers from large amounts of missing data. Methods that use surface-based priors rely on local surface estimates and perform well only when filling small holes. When the holes become large, the reconstruction problem becomes severely under-constrained, which necessitates the use of additional reconstruction priors. In this paper, we introduce weak volumetric priors which assume that the volume of a shape varies smoothly and that each point cloud sample is visible from outside the shape. Specifically, the union of view-rays given by the scanner implicitly carves the exterior volume, while volumetric smoothness regularizes the internal volume. We incorporate these priors into a surface evolution framework where a new energy term defined by volumetric smoothness is introduced to handle large amount of missing data. We demonstrate the effectiveness of our method on objects exhibiting deep concavities, and show its general applicability over a broader spectrum of geometric scenario.
\end{abstract}

\section{Introduction}

Surface reconstruction from incomplete point scans is a difficult and inherently ill-posed problem, requiring geometric priors to guide reconstruction away from available data. Large amount of missing data are primarily caused by selfocclusions, which frequently occur in objects possessing deep concavities, such as the vase in Figure 1. In practice, these kinds of objects are difficult to acquire digitally using common laser scanners: the concavities, especially deep ones, are hard to reach even by a hand-held scanner.

Most priors adopted to handle missing data in surface reconstruction depend essentially on local surface estimation near available data. Positional and normal information at point samples are taken into account to complete data gaps by means of a smooth surface interpolant [CBC $\left.{ }^{*} 01, \mathrm{KBH} 06\right]$. Such approaches are well-suited to fill small holes, but still leave the reconstruction problem under-constrained when large amount of data are missing. In such cases, topological ambiguities often cannot be resolved by smoothly interpolating local surface estimates. Furthermore, over-fitting on sparse data can result in unnatural reconstruction. Surfacebased priors do not fully exploit available data beyond local smoothness measures. In particular, they overlook volumetric properties of the reconstructed shapes.

In this paper, we introduce weak volumetric priors to allow effective digital acquisition of objects exhibiting deep concavities. Our algorithm is specifically designed to handle the large amount of missing data caused by these concavities. Volume-aware surface reconstruction provides a more global view and permits better use of available data. Our priors exploit knowledge about the data acquisition process and a natural volumetric shape property that the local thickness of the shape varies smoothly. They impose external and internal forces which together drive the reconstruction.

- First, points obtained via laser scanning must be visible from the scanner head. By assuming that the scanner head only moves outside the convex hull of the captured shape, we infer that each ray from an acquired point to the scanner head, which we call a view ray, must lie entirely outside the shape. We use these rays to complement available point data, where the rays collectively and conservatively constraint the exterior volume of the shape.

- Second, we consider the interior volume of the shape from 

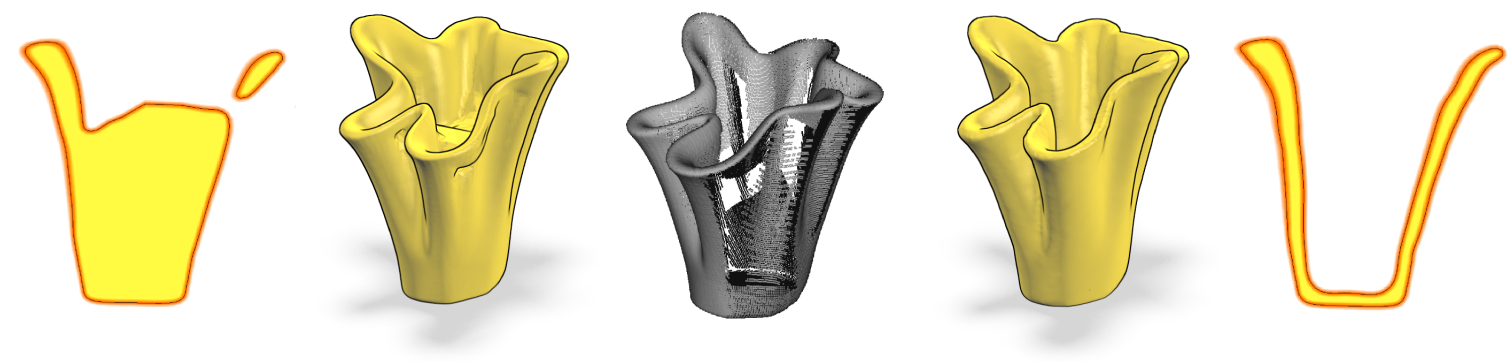

Figure 1: Volume-aware surface evolution or VASE at work (middle right) when reconstructing a concavity-filled vase model with large amounts of missing data (middle). Note the ability of our reconstruction scheme to carve deeply into the concavity of the vase and to regularize its volume, in contrast to Poisson reconstruction (middle left). Two images on the side show cross-sectional views of the respective reconstructions.

the perspective of its medial axis and make a natural assumption that the magnitudes of the radii along the medial axis vary smoothly. Internal forces exerted by such a volumetric regularization can simultaneously influence regions that are far apart along the surface. This key characteristic of the volumetric smoothness prior allows a more global utilization of available data, in contrast to the local nature of surface-based priors. For example, a large gap can be filled correctly by data available from the opposite side of the medial axis.

We implement the two priors within a surface evolution framework, which we call volume-aware surface evolution or VASE. Specifically, we present a level-set formulation [OS88] where the reconstructed surface is the steadystate solution of an interface motion problem. The algorithm is initialized with a conservative bounding box of the input point cloud. The subsequent evolution of the interface surface moves it towards the sampled data with its motion controlled by three combined forces (see Figure 2):

- Data fitting: This external force serves to bring the evolving surface close to the available point samples. View rays from the samples intersect the current surface and each intersection point is moved along the view-ray direction by an amount proportional to the distance between the point sample and the intersection point. We call this process implicit carving as it resembles traditional space carving [CL96, Whi98,SSZCO10], but uses the implicit evolving surface that is controlled by the piercing view rays to carve the exterior volume.

- Surface smoothness: This internal force diffuses the interface surface by minimizing the bi-Laplacian energy. We choose this method over the more frequently used mean curvature flow due to the sparsity of available data. Mean curvature flow performs area minimization and, with sparse point constraints, tends to generate undesirable artifacts near the sample locations.

- Volumetric smoothness: This second internal force diffuses, via a Laplacian formulation, the distribution of radii over the medial axis of the evolving interface surface. Throughout the surface evolution, we maintain and update an approximation of the corresponding medial axis. The medial axis does not explicitly reconstruct the surface; it only serves to define the volumetric regularizer.

We demonstrate the effectiveness and general applicability of VASE on synthetic and real point scans including those of objects containing deep concavities. Comparisons are made to Poisson reconstruction [KBH06], which exhibits generally superior handling of missing data over other methods.

\section{Related work}

Techniques for surface reconstruction from point clouds can be divided into explicit and implicit approaches. Explicit methods construct surfaces by triangulating the point samples directly, often based on Delaunay triangulations [ABK98, DG06, CG06, Dey11]. While able to guarantee correctness provided sampling conditions are met, these methods are unable to deal with significant missing data. Implicit methods [HDD* $92, \mathrm{CBC}^{*} 01, \mathrm{KBH} 06, \mathrm{AC}-$ STD07, NOS09] first construct an implicit function from the point data and then extract the surface by triangulating the function's zero level-set. They are able to handle missing data using a smooth interpolant computed from local surface estimates. Such surface smoothness priors are only suitable to fill small holes in the data.

Visibility priors have been used to compensate for missing data by using view ray directions to carve the exterior volume. Curless and Levoy [CL96] were the first to use such information in range scans where the view rays serve to eliminate exterior voxels. Davis et al. [DMGL02] take an implicit surface evolution approach and apply a surface diffusion kernel to reduce artifacts in transition areas. Recent work by Shalom et al. [SSZCO10] carves large parts of the outside space using continuous cones. However, instead of anchoring the cones by the view rays explicitly, they use maximal cones empty of point samples and produce additional offsurface points based on signed distances to these cones to 


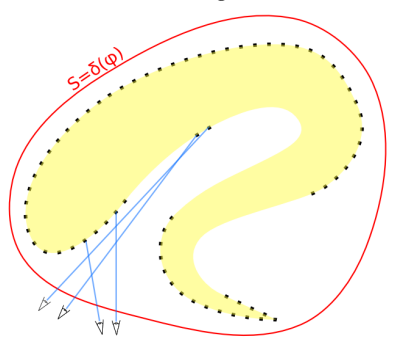

(a)

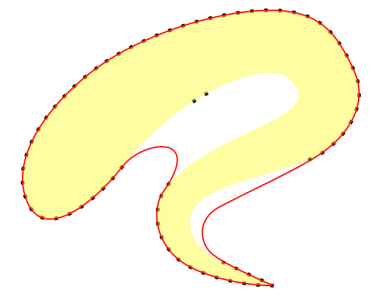

(b)

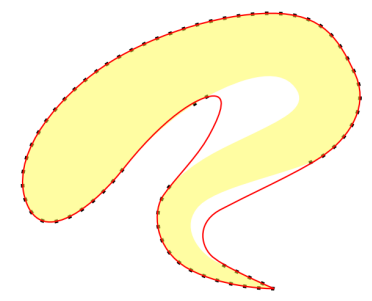

(c)

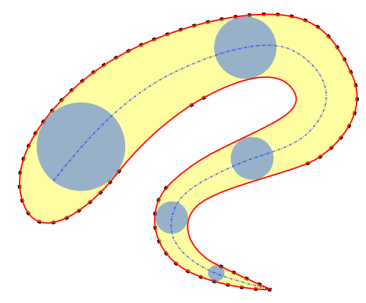

(d)

Figure 2: Overview of VASE for surface reconstruction. Across the three sub-figures the ground truth is shaded in yellow, the scanned samples in black, the active surface in red and the medial axis is represented by a blue dotted curve. (a) An illustration of the initialization of the evolving surface which contracts over the view data samples. (b) Reconstruction achieved by classical methods where only point constraints and smoothness are enforced; samples deep within the concavity are misinterpreted as noise and ignored. (c) The effect of our external view direction constraints. (d) VASE: The combined effect of view directions and volume diffusion.

steer a radial basis function construction. Our algorithm incorporates view rays in a surface evolution framework to implicitly carve the exterior volume. This scheme is similar to the early approach of Whitaker [Whi98] which is also based a level-set formulation but applied to range images.

Tagliasacchi et al. [TZCO09] adopt a non-local volumetric prior which assumes that the reconstructed shape is locally cylindrical everywhere. They rely on rotational symmetry about extracted curve skeletons to compensate for significant missing data. Our algorithm does not need the cylindrical prior; instead, it uses the weaker assumption of volumetric smoothness about the medial axis.

There is a rich literature on level-set based surface evolution for surface reconstruction [OF02]. Ballooning techniques, e.g., [CC93, SLS* 06], start the evolution from inside the reconstructed shape while others, e.g., [EBV05], evolve an active surface via shrinking from an initialization outside the input data. The surface energy that drives the evolution accounts for data fitting and surface regularization, where the latter is typically realized by mean curvature flow [ZOF01] and aims for area minimization. Surface smoothness measures such as Laplacian and bi-Laplacian energies have been widely applied to mesh fairing [BO03]. Our work introduces an additional energy term given by volumetric smoothness over the medial axis to regularize the reconstruction when a large amount of data is missing.

In the context of surface reconstruction from volumetric images, the usefulness of medial-based representations, which follow the internal and volumetric geometry of the object, has been demonstrated on data suffering from noise and weak boundaries [SP08]. However, this line of work has not been applied to surface reconstruction from points clouds with significant missing data.

\section{Overview and formulation}

Here we motivate our approach with a mathematical formulation and first description of VASE. While we can construct our method in a purely implicit way, for efficiency reasons our implementation alternates between a multiscale levelset solver and a mesh-based smoothing step. We first give a high-level description of our method, then provide more detailed formulations of the constraints involved. We discuss individual steps of the implementation in later sections.

Overview We define the reconstruction as the steady state solution to an interface motion problem using the levelset technique of [OS88]. Our input consists of a set of scanned samples $\mathcal{P}=\left\{\mathbf{p}_{\mathbf{i}}, i=1 \cdots N\right\}$ with a unit-length view vector at each sample; we do not require surface normals. We represent the evolving 2-manifold as the zero crossing $\mathcal{S}_{\phi}$ of an implicit function $\phi$ in $3 \mathrm{D}$.

The algorithm is initialized by setting $\mathcal{S}_{\phi}^{t=0}$ to be a scaled bounding box of the input samples, where $t$ indicates iteration count. $\mathcal{S}_{\phi}$ evolves according to a set of forces that modify its geometry and topology by minimizing the following energy:

$$
E=\omega_{1} E_{\mathrm{fit}}+\omega_{2} E_{\mathrm{smooth}}+\omega_{3} E_{\mathrm{vol}} .
$$

Intuitively, we want our resulting surface to fit the data while respecting the visibility prior from the input scan, minimizing $E_{\text {fit }}$. In order to solve the highly under-conditioned problem created by large portions of missing data, we employ both a surface smoothness prior $E_{\text {smooth }}$ as well as a volumetric smoothness prior $E_{\mathrm{vol}}$. The relative strength of these forces is balanced by a tuple $\omega_{i}$ of weights.

The motion of the interface is determined by gradient descent on Eq. (1) and is expressed by forces which act orthogonally to the active surface:

$$
\dot{\mathcal{S}}+\left[\omega_{1} F_{\text {fit }}+\omega_{2} F_{\text {smooth }}+\omega_{3} F_{\text {vol }}\right] \mathbf{n}=0 .
$$

As the interface motion in (2) takes place along the normal of the implicit surface $\mathcal{S}_{\phi}$ and the function $\phi$ is kept as a signed distance field $(\|\nabla \phi\|=1)$, we can rewrite the above expression in a level-set fashion:

$$
\dot{\phi}+\omega_{1} F_{\text {fit }}+\omega_{2} F_{\text {smooth }}+\omega_{3} F_{\text {vol }}=0 .
$$


In practice, we do not express these constraints in terms of a single scalar field. Instead, we represent the input $\mathcal{P}$ by a pair of scalar functions: a density function $\rho_{\mathcal{P}}$ and a viewdistance function $v_{\mathcal{P}}$. These are computed once and, in the first iteration, $v_{\mathcal{P}}$ is used to apply $F_{\text {fit }}$ to a signed distance field $\phi^{t=0}$ in a multiscale level-set solver using mean curvature flow. This computes an implicit surface $\mathcal{S}_{\phi}^{t}$

To apply surface and volumetric constraints, we extract a level set from $\phi^{t}$ as the mesh $\mathcal{S}_{\mathcal{M}}^{t}$, and compute $F_{\text {smooth }}$ by bi-Laplacian smoothing. Once the volume of $\mathcal{S}_{\mathcal{M}}$ has stabilized, we approximate its medial surface by computing its Voronoi poles and compute a smoothed version of its radius function $\tilde{\mathcal{R}}$. From this we are able to compute a signed distance field incorporating $F_{\mathrm{vol}}$. In both cases, we use $\rho_{\mathcal{P}}$ as a weighting function to respect the original data.

We alternate between these two steps, passing the computed fields $F_{\text {smooth }}$ and $F_{\mathrm{vol}}$ from the mesh step back into the levelset solver and performing another iteration to find $\mathcal{S}_{\phi}^{t+1}$, until the surface evolution converges.

Data fitting We minimize the residual distance between $\mathcal{S}^{t}$ and the view-ray measurement as shown later in Figure 3:

$$
E_{\mathrm{fit}}=\sum_{i=1}^{N}\left\|s_{i}^{t}-p_{i}\right\|^{2} .
$$

Following the same derivation provided in [Whi98], it can be shown that this results in the following force field:

$$
F_{\text {fit }}=\delta(\phi) \sum_{i=1}^{N} \varpi\left(x_{i}^{\|}\right) x_{i}^{\|} f\left(x_{i}^{\perp}\right)
$$

where $\delta(\phi)$ is the Dirac function, $f\left(x_{i}^{\perp}\right)$ is a Gaussian function, with kernel width proportional to the sampling density obtained from the scanner, and which is non-zero in spatial locations in proximity of view rays, and $\varpi\left(x_{i}^{\|}\right)$controls the depth of the region behind the sample in which the view ray effects the surface. Where view rays intersect the same voxel, we take a weighted average among the rays as in [CL96], minimizing the least-squares error of the surface approximating the contributing samples.

Surface diffusion To deal with noise and fill small holes, many surface reconstruction algorithms employ surface smoothness as a shape prior. Low-order priors with hard constraints, like those that seek to minimize mean curvature [ZOF01], perform well when dealing with noise and small holes but introduce unpleasant tangential discontinuities when gaps are large. Soft constraints can address this issue but introduce approximation errors. Our solution considers a bi-Laplacian surface smoothness prior, which provides tangential continuity across holes [Lie03]. The bi-Laplacian energy functional is given by:

$$
E_{\text {smooth }}=\int\left\|\Delta_{\mathcal{S}}^{2} S\right\|^{2}
$$

This functional is minimized by the following surface smoothness force [Kob97]:

$$
F_{\text {smooth }}=-\Delta_{\mathcal{S}}^{2} \mathcal{S}=-\Delta_{\mathcal{S}} \kappa,
$$

where $\kappa$ is the mean curvature of the surface. In the level-set domain, forces in the form of Eq. 7 give rise to interface motion known as surface diffusion. As shown in [CS99,Sme03], the discretization of such motion can be quite challenging. We discuss in the following sections how we overcome this problem.

Volume diffusion When the input contains large gaps, the reconstruction problem becomes under-conditioned. Surface-based priors like those described above are sometimes insufficient to reconstruct a reasonable surface. To better constrain the problem, we introduce a novel volumetric prior based on the Medial Axis Transform or MAT $\mathcal{M}, \mathcal{R}=\operatorname{MAT}(\mathcal{S})$. The MAT [SP08] represents the surface as a structure $\mathcal{M}$ composed of sheets which encode local reflectional symmetry, and a radius function $\mathcal{R}$ defined on those sheets. Essentially, $\mathcal{R}$ provides a volumetric representation of the surface; consequently, variations of $\mathcal{R}$ along the medial surface indicate variations in the local volume of the object. Following this intuition, volumetric smoothness is measured by the following energy:

$$
E_{\mathrm{vol}}=\int\left\|\Delta_{\mathcal{M}} \mathcal{R}\right\|^{2} .
$$

The volumetric force associated with this energy can be derived similarly to $E_{\text {smooth }}$ :

$$
F_{\mathrm{vol}}=-\Delta_{\mathcal{M}} \mathcal{R}
$$

Figure 2 illustrates the difference between a reconstruction which only employs surface smoothness as a prior, as in Figure 2(b), and one that also equalizes volume across the shape, as in Figure 2(d). We further demonstrate the effectiveness of this prior in Section 7 on a variety of data.

\section{Density and view-distance field generation}

We precalculate a pair of scalar fields from the original data set to guide our reconstruction algorithm. These are used both to obtain an initial approximation of the full surface and to guide subsequent steps of the algorithm.

\subsection{Density field}

The density field $\rho_{\mathcal{P}}$ is computed from the input by convolution of a Gaussian kernel with the input, producing a dense scalar field which we use to guide later reconstruction steps. In particular, $\rho_{\mathcal{P}}$ influences the bi-Laplacian and volume-diffusion steps described in Sections 6.1 and 6.3 by constraining surface movement where input data is present.

We build $\rho_{\mathcal{P}}$ in the same manner as [KBH06], weighting each element in a volume enclosing $\mathcal{P}$ by the number of adjacent samples using the method described in [Par62]. We 
then convolve the resulting 3D grid with a Gaussian kernel whose variance is proportional to the resolution of $\mathcal{P}$ to produce a smooth representation of sample density.

\subsection{View-distance field}

Each sample in our input has an associated view ray indicating the direction of the scanner head. Here we compute a scalar field $v_{\mathcal{P}}$ in which every voxel intersecting a view ray contains the distance to the sample on that ray. This field pulls the implicit surface $\mathcal{S}_{\phi}$ towards the samples in $\mathcal{P}$ against the influence of $F_{\text {smooth }}$.

We build $v_{\mathcal{P}}$ in a similar manner to $\rho_{\mathcal{P}}$, using a standard 3D-DDA algorithm to encode the view-rays in a 3D grid. Next we populate the

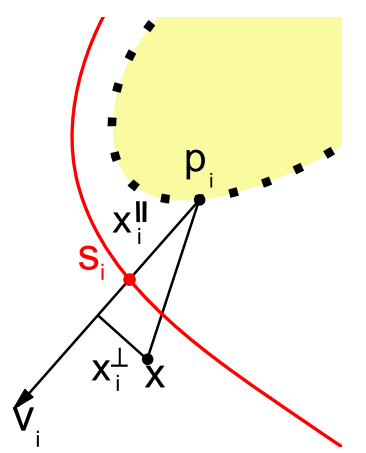

Figure 3: The value of $\mathrm{v}_{\mathcal{P}}$ at a point $x$ is found in terms of the components $x_{i}^{\|}$and $x_{i}^{\perp}$ for each view ray $v_{i}$. full grid by computing a discretized version of Eq. (5) at each node:

$$
F_{\text {fit }}(x)=\sum_{i=1}^{N} \varpi\left(x_{i}^{\|}\right) x_{i}^{\|} f\left(x_{i}^{\perp}\right),
$$

where $\varpi$ is the windowing function used in [Whi98], which prevents view-rays from over-penetrating the shape, and $f$ is a Gaussian as in Section 4.1. The function $F_{\text {fit }}$ is trilinearly interpolated after discretization. We compute $v_{\mathcal{P}}$ over the whole volume; thus, it is not restricted to $\mathcal{S}_{\phi}$ as in Eq. (5).

\section{Multiscale distance field construction}

We apply the computed forces to the surface $\mathcal{S}_{\phi}$ using a multiscale narrow-band level-set solver. We initialize the solver with a trivial surface defined as a band around the bounding box of the input point cloud. The sparse signed distance field is then evolved by mean curvature flow, subject to the forces defined by (10). This procedure contracts the active surface onto the data while respecting the position and orientation of the input samples, thus producing a first approximation of the full surface.

As we are only interested in one particular level set - the zero crossing of $\phi-$ the evolution can be performed efficiently by using a sparse-field method [Whi98]. The method applies motion only to a limited number of voxels of the solver's domain: those containing the zero crossing of the function. Voxels in a small neighborhood near the zero crossing, where $|\phi|<2.5$, are also updated to retain the property that $\|\nabla \phi\|=1$. This permits us to compute curvature measures necessary for the evolution directly from $\phi$.
In order to be able to evolve the surface onto the data efficiently, we perform the contraction operation in a multiscale fashion. Starting with a coarse representation of $\mathcal{S}_{\phi}$, we evolve the implicit function until convergence and we then refine it onto a denser grid by trilinear interpolation. Once $\mathcal{S}_{\phi}$ has converged at the desired resolution, we extract its zero level-set using marching tetrahedrons [BO05] to produce a triangle mesh $\mathcal{S}_{\mathcal{M}}$.

We proceed by applying surface and volume diffusion to $\mathcal{S}_{\mathcal{M}}$ as described in the next section, from which we derive the forces $F_{\text {smooth }}$ and $F_{\text {vol }}$ which will drive $\mathcal{S}_{\phi}$ toward our final desired result. Due to the non-linearity of $E_{\mathrm{vol}}$, we need to perform this operation in an interleaved fashion: by discretizing $\mathcal{S}_{\phi}^{t}$, computing a set of forces on $\mathcal{S}_{\mathcal{M}}^{t}$, and mapping them back into the domain of $\phi$ to compute $\mathcal{S}_{\phi}^{t+1}$.

We choose an interleaved approach for several reasons. Most importantly, computing bi-Laplacian flow directly on $\mathcal{S}_{\phi}$ is unusually difficult, and the mean curvature flow method described above would not suffice as it does not allow us to obtain tangential continuity. In terms of efficiency, the medial axis can be more quickly computed on an explicit representation as its complexity is $O(n \log n)$, where $n$ is the total number of voxels in the domain in one case and the number of vertices in the other; this last quantity corresponds to only the number of voxels intersecting the surface. Finally, an explicit representation permits us to achieve sub-pixel accuracy in the positioning of the Voronoi loci, which a discrete representation of the medial axis would not provide.

\section{Surface and volume diffusion}

Having extracted the zero level set of our implicit surface $\mathcal{S}_{\phi}^{t}$ as a triangle mesh $\mathcal{S}_{\mathcal{M}}^{t}$, we next compute the surface and volume smoothness constraints directly from $\mathcal{S}_{\mathcal{M}}^{t}$ to produce representations for $F_{\text {smooth }}^{t}$ and $F_{\text {vol }}^{t}$. These forces are returned to the level-set solver and applied to produce $\mathcal{S}_{\phi}^{t+1}$.

Our surface-based diffusion computations most strongly differentiate our approach from that of Whitaker in [Whi98]. Since we do not assume any structure in the view-ray data, having laser scans rather than range images, a straightforward implementation of his approach is computationally impractical. Furthermore, our volumetric smoothness prior, not easily calculated in an implicit setting, permits us to reconstruct a variety of shapes with significant missing data.

Note that until our initial approximation obtained by mean curvature flow has converged, it is unlikely that $\mathcal{S}_{\mathcal{M}}^{t}$ contains the information necessary for the enforcement of tangential boundary constrains for the bi-Laplacian solver. Similarly, the MAT of $\mathcal{S}_{\mathcal{M}}^{t}$ is unlikely to contain useful information before convergence. Hence, until $\phi$ is in the process of converging towards the data points in $\mathcal{P}$, we omit the computation of $F_{\text {vol }}^{t}$ or $F_{\text {smooth }}^{t}$. 


\subsection{Bi-Laplacian smoothing}

In order to produce a surface without the tangential discontinuities inherent in mean curvature flow, we apply biLaplacian smoothing [SCOIT05] to $\mathcal{S}_{\mathcal{M}}$ to compute a new smoother surface $\tilde{\mathcal{S}_{\mathcal{M}}}$, which we then use to encode the smoothness force $F_{\text {smooth. }}^{t}$. Rather than treat each component of $\mathcal{S}_{\mathcal{M}}$ equally, however, we respect the position of the input data and control the evolution of the vertices of $\mathcal{S}_{\mathcal{M}}$ using the density field $\rho_{\mathcal{P}}$. The density field allows us to specify boundary constraints, allowing parts of $\mathcal{S}_{\mathcal{M}}$ that are far from the scanned data to move freely while restraining those that are close to elements in $\mathcal{P}$. Here we simply use the value of $\rho_{\mathcal{P}}$ at a vertex as the constraint weight in our Laplacian operator. The smoothed surface can then simply be converted into the volumetric force $F_{\text {smooth }}^{t}$ by constructing its associated signed Euclidean distance field in the domain defined by the level-set solver. We also use this distance field $F_{\text {smooth }}^{t}$ to encode $F_{\mathrm{vol}}^{t}$ as described later.

\subsection{Voronoi construction of medial axes}

As noted in Section 3, the MAT of a surface provides an elegant description of the variation of its internal volume. We approximate the MAT of $\mathcal{S}_{\mathcal{M}}^{t}$ by constructing its medial axis representation $\mathcal{M}_{\mathcal{S}}^{t}$ from its Voronoi diagram $\operatorname{Vor}_{\mathcal{S}}^{t}$.

We extract $\operatorname{Vor}_{\mathcal{S}}^{t}$ by computing the Voronoi diagram of the vertices of $\mathcal{S}_{\mathcal{M}}^{t}$. Following the ideas in [ABK98], we then associate each vertex on $\mathcal{S}_{\mathcal{M}}^{t}$ with the corresponding Voronoi pole in the interior of the shape; we can exclude exterior poles by checking the value of $\phi^{t}$ at each pole. These poles represent the medial surface $\mathcal{M}_{\mathcal{S}}^{t}$ of $\mathcal{S}_{\mathcal{M}}^{t}$. We do not need to compute the connectivity of $\mathcal{M}_{\mathcal{S}}^{t}$ in order to compute $F_{\text {vol }}$.

As constructed, $\mathcal{M}_{\mathcal{S}}^{t}$ may have a number of nodes close to $\mathcal{S}_{\mathcal{M}}^{t}$ as the medial axis approaches small features in the surface. We explicitly filter the medial axis when it approaches the surface, setting to zero the weights of nodes within the width of one voxel from $\mathcal{S}_{\mathcal{M}}^{t}$ in the subsequent volume diffusion process. Voronoi loci so close to the surface are not likely to contribute significant information to the MAT.

We next compute the aperture spoke angle $\alpha_{i}$ at each node in $\mathcal{M}_{\mathcal{S}}^{t}$, which we will use to weight the importance of associated vertices in the volume diffusion step. As noted in [DZ02], medial loci with large $\alpha$ contribute more strongly to the MAT, and thus should exert greater influence upon $F_{\text {vol. }}^{t}$. Each node $\mathcal{M}_{\mathcal{S}}^{i} \in \mathcal{M}_{\mathcal{S}}^{t}$ is a Voronoi vertex of a number of vertices $V$ on $\mathcal{S}_{\mathcal{M}}^{t}$. The aperture spoke angle $\alpha_{i}$ of $\mathcal{M}_{\mathcal{S}}^{i}$ is the maximum angle between two edges connecting $\mathcal{M}_{\mathcal{S}}^{i}$ to elements of $V$. We call the two mesh vertices which define $\alpha_{i}$ the spoke vertices of $\mathcal{M}_{\mathcal{S}}^{i}$. These spoke vertices give us an approximation of $\mathcal{R}$ at $\mathcal{M}_{\mathcal{S}}^{i}$, which we use to compute $F_{\text {vol }}^{t}$. Note that this relationship is not bijective: each vertex on $\mathcal{S}_{\mathcal{M}}^{t}$ is associated with a single vertex in $\mathcal{M}_{\mathcal{S}}^{t}-$ its Voronoi pole [ABK98].

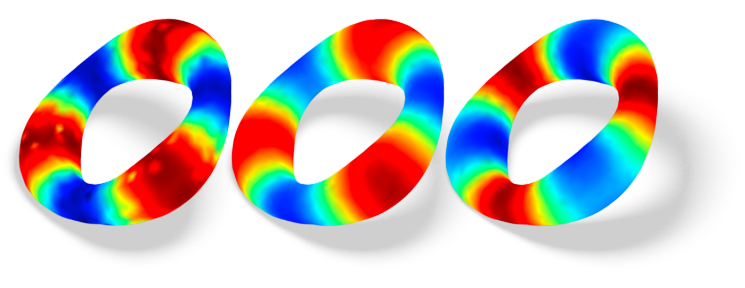

Figure 4: For each vertex of $\mathcal{S}_{\mathcal{M}}$ we display: (a) the radius function $\mathcal{R}$, (b) the filtered radius function $\tilde{\mathcal{R}}$, and (c) the volumetric force which equalizes the volume as $\tilde{\tilde{\mathcal{R}}}-\tilde{\mathcal{R}}$.

\subsection{Volume smoothing}

Having thus obtained an approximation of $\mathcal{R}$ on $\mathcal{M}_{\mathcal{S}}^{t}$, we next compute two smoothed radius functions $\tilde{\mathcal{R}}$ and $\tilde{\tilde{\mathcal{R}}}$ as scalar fields defined on the mesh surface. As shown in Figure $4\left(\right.$ c), the difference $\tilde{\tilde{\mathcal{R}}}-\tilde{\mathcal{R}}$ provides a force field on $\mathcal{S}_{\mathcal{M}}^{t}$ which will attempt to enforce smooth volume variation along the medial axis of $\mathcal{S}_{\mathcal{M}}^{t}$.

As shown in Figure 4(a), the function $\mathcal{R}$ contains a significant amount of noise. To overcome this problem, we perform a first diffusion step, shown in Figure 4(b), where the strength of the constraints is proportional to the spoke aperture angle $\alpha$. Small spoke aperture angles are associated with small surface features which obscure the general volumetric variation of the object. While the spoke angle of a node near the surface may be large, nodes near the surface have already been discarded thus the remaining nodes along a branch corresponding to a noisy feature will be small.

Now that our surface is equipped with a smooth function $\tilde{\mathcal{R}}$, which represents volumetric information, we can express the volumetric force as $F_{\text {vol }}^{t}=\tilde{\tilde{\mathcal{R}}}-\tilde{\mathcal{R}}$, where $\tilde{\tilde{\mathcal{R}}}$ is obtained simply by smoothing $\tilde{\mathcal{R}}$. Here the equivalence to (9) can be established by considering that given a scalar function $f$ one can produce a smoothed version $\tilde{f}$ by removing its local detail, that is, by taking the Laplacian of the function: $\tilde{f}=f-\Delta f$. This force, defined on the mesh surface, is incorporated with $F_{\text {smooth }}^{t}$ as an offset to the signed Euclidean distance field computed in Section 6.1, constructing a new field which incorporates the constraints applied in this section. We generate a new force field $f_{\text {total }}^{t}(x)=F_{\text {vol }}^{t}\left(x_{S}\right)+\left\|x-x_{S}\right\|$, where $\|\cdot\|$ is a signed Euclidean distance and $x_{S}$ is the closest point on $\tilde{S}_{\mathcal{M}}$ to $x$. We pass this composite distance field to the solver from Section 5 to enable computation of $\mathcal{S}_{\phi}^{t+1}$.

\section{Results}

In this section, we show results of surface reconstruction using VASE. The synthetic point datasets used in Figures 8 and 9 were taken from the work of Shalom et al. [SSZCO10]. The raw point scans in Figures 1, 5, 6, and 7 were obtained using a Polhemus Cobra hand-held scanner. Each object was scanned under typical acquisition settings, with algorithm parameters being set according to scanner resolution. However, due to the presence of deep concavities in the three 

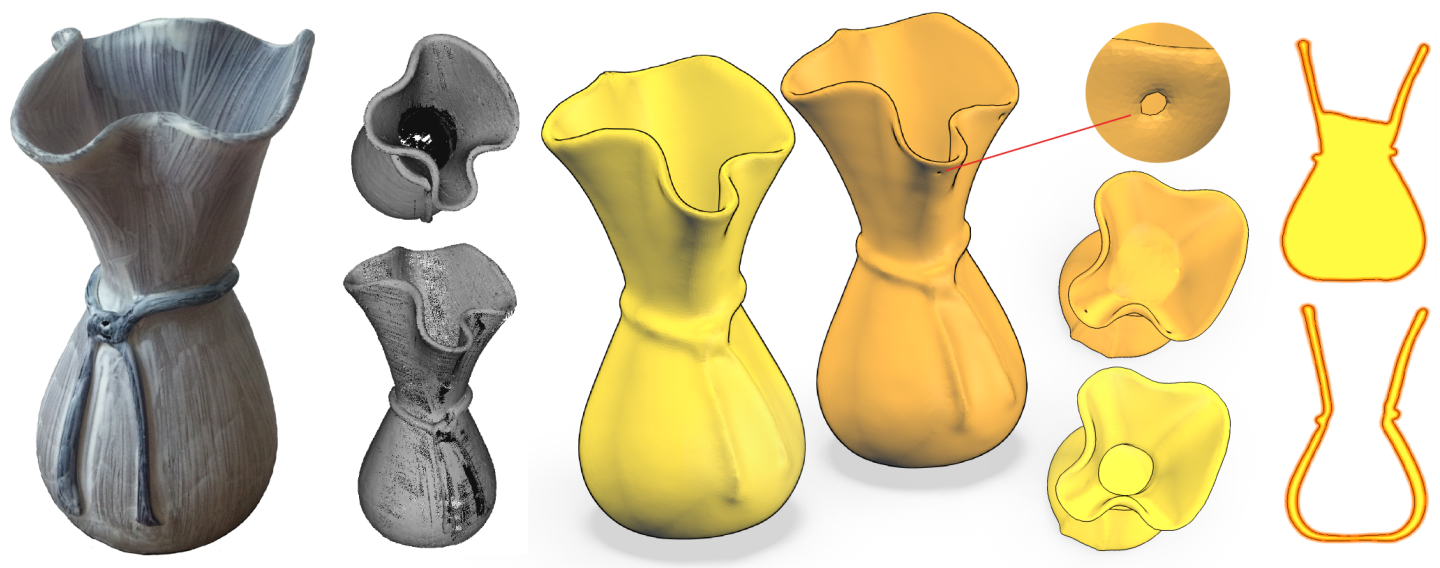

Figure 5: Results of VASE (yellow) and Poisson (orange) on a scan of a tapered vase. The prior used by Poisson leads to a truncation of the concavity before reaching the vase's taper, while VASE enables the recovery of the whole interior. Poisson also erroneously preserves gaps in the scan as manifested by small holes in the reconstructed surface.
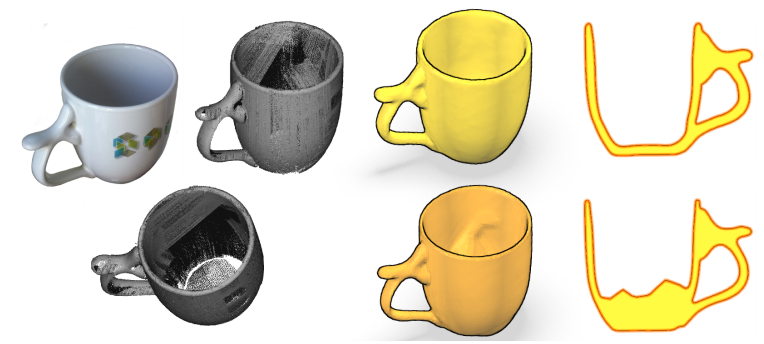

Figure 6: Reconstruction of a coffee cup with non-zero genus. Our method successfully recovers the shape's variations in interior volume while accurately reconstructing the interior of the cup.

container-type objects, a large amount of data is missing in the objects' interiors. To obtain the view rays, we modified the scanner interface software as instructed by the vendor.

In all the examples shown, we compare the results from VASE to those from Poisson reconstruction. We note that Poisson reconstruction requires normals at the point samples, estimated by applying PCA to a local neighbourhood, but VASE does not. Over areas representing deep concavities of an object, our method outperforms Poisson due to the effect of the volumetric priors. In addition, the volumetric smoothness prior avoided the creation of small holes over the tapered vase (Figure 5) and led to better reconstruction near the knee of the jazzman model (Figure 7). As algorithm behaviour varies roughly linearly between that shown in Figure 2(c) and (d) when parameters not directly related to scanner resolution are varied, these parameters can be chosen in a principled way.

Over other areas of the acquired objects, we do not observe noticeable differences between results produced by the two reconstruction methods; VASE performs as well as the state-

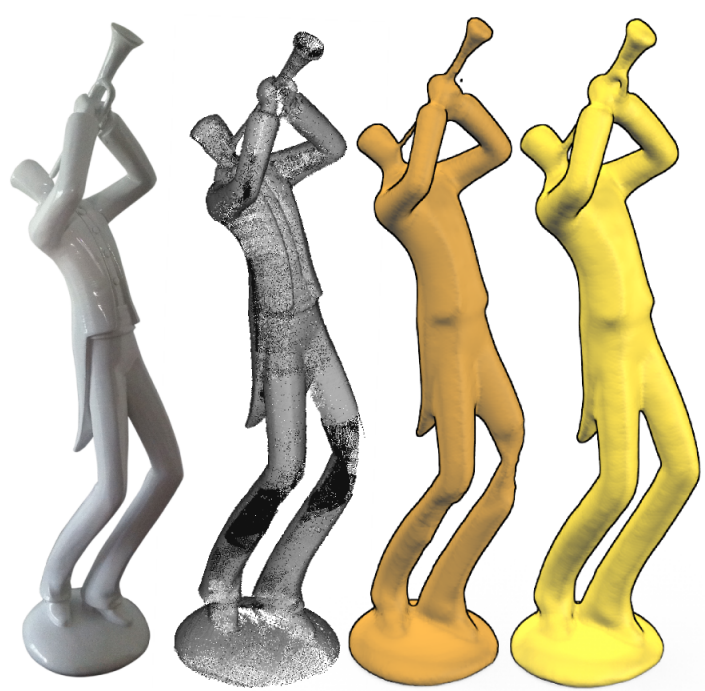

Figure 7: Reconstruction of a stylized figure. Our volumetric priors allow us to reconstruct the knee and trumpet more faithfully. Variations in the interior volume $(\mathcal{R})$ are handled without error, though sharp edges are not fully preserved.

of-the-art Poisson reconstruction, even on models with nonzero genus as in Figures 6 and 9. Our ability to reconstruct correct topology depends upon the view rays reported by the scanner; note that in Figure 9 our volumetric prior is able to preserve holes in the input, which are discarded by Poisson reconstruction.

Figure 10 shows the evolution of $\mathcal{S}$ over time. Note the way the samples in the central channel pull $\mathcal{S}$ towards them (and contrast this with the result of Poisson reconstruction in Figure 8), and the regularized volume in the final cross-section. Both of these features reflect our novel volumetric priors. 

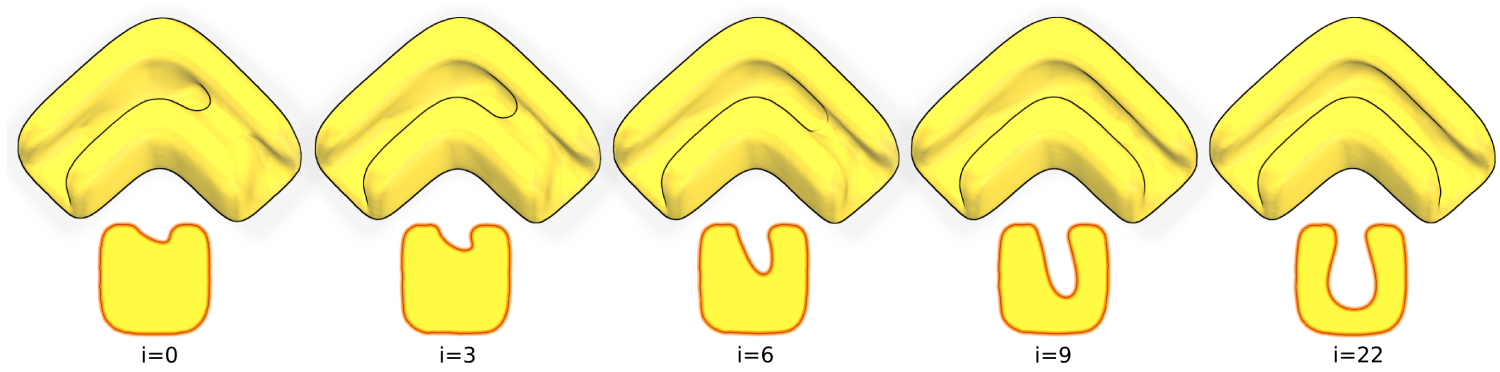

Figure 10: The evolution of $\mathcal{S}$ over time on the "shyel" dataset of Figure 8. Cross-sections of the right-hand segment are also shown. The end result exhibits correct structure and volumetric smoothness.

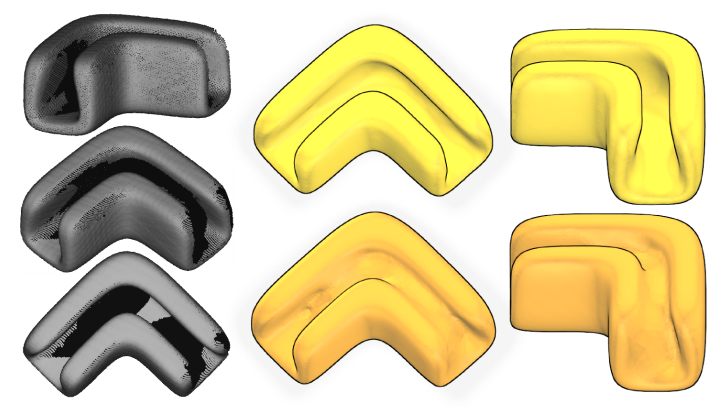

Figure 8: The "shyel" mechanical piece is virtually scanned (left). The result achieved by VASE (top right, in yellow) correctly reconstructs the central channel, while that from Poisson (bottom right, in orange) both ignores the small set of samples within that channel and is unable to use volumetric priors to compensate for the large amount of missing data.

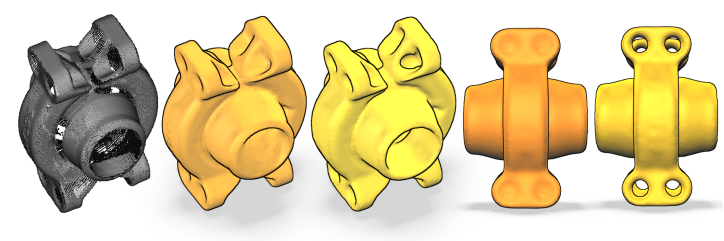

Figure 9: Surface reconstruction on a machine part. Note that our volumetric priors preserve small and large holes, which are filled by Poisson reconstruction.

We have implemented VASE in a combination of MATLAB and $\mathrm{C}++$, yielding runtime performance of roughly five minutes to reconstruct the scans shown in this paper using a maximum grid size of $200^{3}$ in the multiscale level-set solver. One of the bottlenecks in our current implementation is zero level-set extraction: the computation of the bi-Laplacian and volumetric smoothness forces requires a conversion from the implicit representation to an explicit mesh representation. Subsequently, the medial axis needs to be re-computed. The required conversion between implicit and explicit representations of $\mathcal{S}$ increases runtime to the point that comparing efficiency to existing techniques is not informative; however, we present a possible avenue for significant performance improvement in future work.

\section{Conclusion and future work}

We introduce VASE, a surface reconstruction method built around novel volumetric priors for visibility and interior volume smoothness. These priors are specifically designed to handle highly incomplete point scans, which are typically the results of scanner capture of objects with deep concavities. VASE is generally applicable to a broader class of objects. However, for scans with only small amount of missing data, other state-of-the-art methods such as Poisson reconstruction already offer efficient and effective solutions. As a diffusion-based surface evolution, VASE is designed to reconstruct smooth shapes and does not strive to preserve or enhance sharp features, e.g., for certain CAD models.

One limitation of our current approach is its dependence upon view-ray information when constructing the topology of the medial axis; our reconstruction priors are designed for geometry inference. When the problem is severely underconstrained, correct topology inference may inevitably require user assistance [SLS*07]. Consequently, it might be helpful to allow the user to identify areas interactively from which the surface evolution would grow into a concave region of the object.

Another limitation lies in our discretization of the volumetric force. While our formulation of the surface evolution energy implies a diffusion over the medial axis, we currently perform diffusion on the surface. This avoids explicit tracking of the topology of the medial axis, which may become complex. While this choice has not caused problems on the tested models, it is a potential source of error as high-frequency surface details are removed as noise. Hence, our method performs best on shapes whose local thickness values vary smoothly. We would like to investigate the magnitude of this error and consider alternative solutions.

Finally, the major practical limitation is speed. We note in Section 7 that level-set meshing and medial axis recomputation form a significant performance bottleneck. This bottleneck may be lifted if we resort to a fully explicit formula- 
tion, calculating all forces on the mesh rather than within an implicit level-set solver. This would permit us to update the Voronoi diagram and thus the medial axis during the interface motion, pre-compute the Laplacian matrices for surface smoothing at each iteration, and eliminate a number of parameters. Normal flows for explicit mesh representations can be done by extending the work of [PB07] which creates an adaptive discretization of the domain for surface evolution. A 3D implementation of this method was recently proposed in [DP10] for reconstruction from range scans. We wish to investigate these improvements in future work.

\section{References}

[ABK98] Amenta N., Bern M., Kamvysselis M.: A new Voronoi-based surface reconstruction algorithm. In Proc. SIGGRAPH (1998), ACM, pp. 415-421. 2, 6

[ACSTD07] Alliez P., Cohen-Steiner D., Tong Y., DesBRUN M.: Voronoi-based variational reconstruction of unoriented point sets. In Proc. Symp. on Geom. Processing (SGP) (2007), pp. 39-48. 2

[BO03] BelyaeV A., OHTAKe Y.: A comparison of mesh smoothing methods. In Proc. Israel-Korea Bi-National Conf. on Geom. Modeling and Comp. Graphics (2003), pp. 83-87. 3

[BO05] Boissonnat J.-D., OUdot S.: Provably good sampling and meshing of surfaces. Graphical Models 67 (2005), 405-451. 5

[CBC*01] Carr J. C., Beatson R. K., Cherrie J. B., Mitchell T. J., Fright W. R., McCallum B. C., Evans T. R.: Reconstruction and representation of $3 \mathrm{D}$ objects with radial basis functions. In Proc. SIGGRAPH (2001), pp. 67-76. 1, 2

[CC93] Cohen L. D., CoHEN I.: Finite-element methods for active contour models and balloons for 2D and 3D images. IEEE Trans. Pat. Ana. \& Mach. Int. (PAMI) 15, 11 (1993), 1131-1147. 3

[CG06] Cazals F., Giesen J.: Delaunay triangulation based surface reconstruction. In Effective Computational Geometry for Curves and Surfaces. Springer, 2006, pp. 231-276. 2

[CL96] CuRLESS B., LEVOY M.: A volumetric method for building complex models from range images. In Proc. SIGGRAPH (1996), pp. 303-312. 2, 4

[CS99] Chopp D. L., SETHIAN J. A.: Motion by intrinsic laplacian of curvature. Interfaces and Free Boundaries 1 (1999), 1-18. 4

[Dey11] DEY T. K.: Curve and Surface Reconstruction: Algorithms with Mathematical Analysis. Cambridge, 2011. 2

[DG06] DEY T. K., Goswami S.: Provable surface reconstruction from noisy samples. Comput. Geom. Theory Appl. 35, 1-2 (2006), 124-141. 2

[DMGL02] Davis J., Marschner S., GARr M., LeVoy M.: Filling holes in complex surfaces using volumetric diffusion. In Symp. 3D Data Proc. Vis. Trans. (2002). 2

[DP10] Delaunoy A., Prados E.: Gradient Flows for Optimizing Triangular Mesh-based Surfaces: Applications to 3D Reconstruction Problems dealing with Visibility. International Journal of Computer Vision (2010), 1-24. 9

[DZ02] DEY T., ZHAO W.: Approximating the medial axis from the voronoi diagram with a convergence guarantee. In Proc. Euro. Symp. Algorithms (2002). 6
[EBV05] Esteve J., Brunet P., Vinacua À.: Approximation of a variable density cloud of points by shrinking a discrete membrane. Computer Graphics Forum 24, 4 (2005), 791-808. 3

[HDD*92] Hoppe H., DeRose T., Duchamp T., McDonald J., Stuetzle W.: Surface reconstruction from unorganized points. In Proc. SIGGRAPH (1992), pp. 71-78. 2

[KBH06] Kazhdan M., Bolitho M., Hoppe H.: Poisson surface reconstruction. In Proc. Symp. on Geom. Processing (SGP) (2006), pp. 61-70. 1, 2, 4

[Kob97] Kobbelt L.: Discrete Fairing. In Proceedings of the Seventh IMA Conference on the Mathematics of Surfaces (1997), vol. 97, pp. 101-131. 4

[Lie03] LIEPA P.: Filling holes in meshes. Computer Graphics Forum (Proc. EUROGRAPHICS) 30, 6 (Dec. 2003), 200-205. 4

[NOS09] NAGaI Y., OHTAKE Y., SUZUKI H.: Smoothing of partition of unity implicit surfaces for noise robust surface reconstruction. In Proc. Symp. on Geom. Processing (SGP) (2009), pp. 1339-1348. 2

[OF02] Osher S., FEDKIW R.: Level Set Methods and Dynamic Implicit Surfaces. Springer, 2002. 3

[OS88] OSHER S., SETHIAN J.: Fronts propagating with curvature-dependent speed: algorithms based on HamiltonJacobi formulations. Journal of computational physics 79, 1 (1988), 12-49. 2, 3

[Par62] PARZEN E.: On estimation of a probability density function and mode. In Ann. Math Stat. (1962), vol. 33, pp. 10651076. 4

[PB07] Pons J.-P., BoIssonnAT J.-D.: A lagrangian approach to dynamic interfaces through kinetic triangulation of the ambient space. Computer Graphics Forum 26, 2 (June 2007), 227-239. 9

[SCOIT05] Sorkine O., COHEN-OR D., Irony D., Toledo S.: Geometry-aware bases for shape approximation. IEEE transactions on visualization and computer graphics 11, 2 (2005), 171-80. 6

[SLS*06] Sharf A., Lewiner T., Shamir A., Kobbelt L., COHEN OR D.: Competing fronts for coarse-to-fine surface reconstruction. Computer Graphics Forum (Proc. EUROGRAPHICS) 25, 3 (2006), 389-398. 3

[SLS*07] Sharf A., Lewiner T., Shklarski G., Toledo S., COHEN-OR D.: Interactive topology-aware surface reconstruction. ACM Trans. Graph. 26, 3 (2007), 43:1-43:10. 8

[Sme03] SMEREKA P.: Semi-implicit level set methods for curvature and surface diffusion motion. Journal of Scientific Computing 19 (2003), 439-456. 4

[SP08] SIDDIQI K., PIZER S.: Medial representations: mathematics, algorithms and applications. Springer, 2008. 3, 4

[SSZCO10] Shalom S., Shamir A., Zhang H., Cohen-OR D.: Cone carving for surface reconstruction. ACM Trans. on Graphics (Proc. SIGGRAPH Asia) 29, 5 (2010), 150:1-150:10. 2,6

[TZCO09] TAGLiASACCHI A., ZHANG H., COHEN-OR D.: Curve skeleton extraction from incomplete point cloud. ACM Trans. Graph. 28, 3 (2009), 71-1, 71-9. 3

[Whi98] WhITAKeR R. T.: A level-set approach to 3D reconstruction from range data. International Journal of Computer Vision 29, 3 (1998), 203-231. 2, 3, 4, 5

[ZOF01] ZHAO H., Osher S., FEDKIW R.: Fast Surface Reconstruction Using the Level Set Method. In VLSM (2001), Published by the IEEE Computer Society, p. 194. 3, 4 